\section{CLOSE TO THE EDGE}

Snowflakes are often seen as a paradigm of complexity emerging from simple rules. But in fact the rules are far from simple: they represent an interplay of effects at length scales ranging from the molecular to the macroscopic.

The subtlety of this crystal-growth process becomes apparent from the diversity of morphologies it generates with only slight changes in humidity or temperature. Snowflakes are not always the familiar, intricate six-pointed dendrites: they might also be hexagonal plates or prisms, needles, plates with triangular symmetry, or rather irregular. Ukichiro Nakaya, a pioneer of laboratory-grown snowflakes, memorably called them "letters sent to us from the sky" - but we still don't fully understand how to read them.

The usual explanation for the sixfold dendritic shape invokes a balance between dendritic growth through the Mullins-Sekerka instability and the anisotropy of crystal surface free energy due to the underlying ice lattice. Only in sixfold directions can needle-like growth outrun the tip-splitting instability that would induce disorder ${ }^{1}$. Why, though, this shifting of morphology between columns and plates, ramifications and facets?

In particular, the persistence of columns and plates at relatively low humidity, and transitions between them as the temperature is lowered, are puzzling. At around $-16^{\circ} \mathrm{C}$ the interface between the prismatic face of bulk ice and water undergoes a roughening transition in which fluctuations in the height of the solid surface diverge. This should round the crystal planes and erode facets, and so raises the question of how snowflakes shaped like faceted plates and columns survive close to freezing point.

The surface of ice is itself more complex than some models of snowflake formation acknowledge. Both simulations and experiments indicate that, close to but below the melting point, the surface supports a thin liquid-like layer ${ }^{2}$ : a notion that dates back to Michael Faraday, and which has been invoked to explain the low friction of ice. This water layer arises from a premelting transition.

Benet et al. now suggest that this quasi-liquid layer might explain the growth morphologies of ice crystals in the atmosphere ${ }^{3}$. Their computer simulations of a thin film of ice close to water's triple point $\left(0.01{ }^{\circ} \mathrm{C}\right)$ show that the first few molecular layers are melted. They model this film in terms of the two interfaces bounding it: the liquid-solid and liquid-vapour surfaces. Crystal growth seems to depend sensitively on how these interfaces 'feel' one another.

Benet and co-workers find that below about $-2{ }^{\circ} \mathrm{C}$ the coupling of interfaces that occurs more or less regardless of the (finite) thickness of the quasi-liquid layer has the

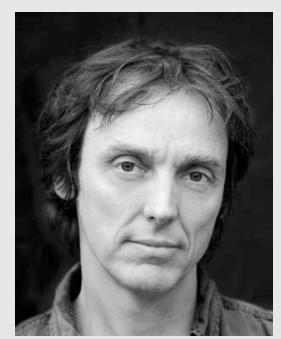

PHILIP BALL

effect of suppressing long-wavelength roughness in the ice-water interface of the prismatic plane. So below this temperature the solid surface stays macroscopically smooth and crystal growth is slowed down.

A role of the premelted layer in ice-crystal growth morphology was proposed in 1982 as an explanation for switches between columnar and plate forms $s^{4}$. Could the new results account for these too? That depends on the relative growth rates of the prismatic and basal crystal faces. The former is enhanced due to roughening above $-2{ }^{\circ} \mathrm{C}$; preliminary data suggest the latter is not, and so some kind of crossover might take place. But bigger simulations will be needed to check.

References

1. Ben-Jacob, E., Goldenfeld, N., Langer, J. S. \& Schön, G. Phys. Rev. Lett. 51, 1930 (1983).

2. Dash, J. G., Rempel, A. W. \& Wettlaufer, J. S. Rev. Mod. Phys. 78, 695 (2006).

3. Benet, J., Llombart, P., Sanz, E. \& MacDowell, L. G. Phys. Rev. Lett. 117, 096101 (2016).

4. Kuroda, T. \& Lacmann, R. J. Cryst. Growth 56, 189-205 (1982). 